\title{
KORNABTROCKNUNG AUF DEM HALM UND WITTERUNG
}

\author{
W. RENTSCHLER und V. VOIGT \\ (vorgetragen von W. Rentschler)
}

Institut für Physik und Meteorologie der Landwirtschaftlichen Hochschule Hohenheim, Deutschland

\section{ZUSAMMENFASSUNG}

\begin{abstract}
Anlässlich von Untersuchungen über den Mähdreschereinsatz wurden Messungen über den Zusammenhang zwischen dem Witterungsablauf und der Feuchte des Getreidekorns auf dem Halm durchgeführt. Es ergab sich, dass auch für diesen Fall mit genügender Näherung das 14 Uhr Sättigungsdefizit für die Tagesabtrocknung massgebend ist. Es werden Beziehungen zur Bestimmung der Abtrocknung aus dem Sättigungsdefizit, sowie solche zur Bestimmung der Wasseraufnahme bei Regen gegeben.
\end{abstract}

Der wirtschaftliche Einsatz des Mähdreschers hängt, wie bei keinem andern Ernteverfahren, sehr stark vom Witterungsverlauf während der Ernteperiode ab. Es war daher erwünscht, für das Gebiet der Bundesrepublik Deutschland einen Úberblick über die Einsatzmöglichkeiten dieses Ernteverfahrens zu erhalten.

$\mathrm{Da}$ keine speziellen Beobachtungen aus einer genügenden Zahl von Jahren vorlagen blieb nichts anderes übrig, als Messwerte aus jüngerer Zeit in geeigneter Weise auf einen längeren Zeitraum zu übertragen. Durch das Kuratorium für Technik in der Landwirtschaft wurde zunächst in den Jahren 1952, 1953 und 1954 an fünf Orten der Bundesrepublik der Wassergehalt des auf dem Halm reifenden Getreidekornes, bei Winterweizen, Wintergerste und Sommergerste gemessen (VoIGT).

Die Messungen bestätigten die früheren Ergebnisse (BERG und Oттоson, 1949), dass alle Getreidearten sich hierbei sehr ähnlich verhalten.

Mit einem Aspirationspsychrometer und Thermohygrographen wurden jeweils Lufttemperatur und Luftfeuchtigkeit in zwei Meter Höhe bestimmt, die Niederschlagsmenge wurde in Ahrenhöhe gemessen und die andern interessierenden Elemente subjektiv beobachtet. Abtrocknungs- und Anfeuchtungsperioden wurden nur getrennt auf den Zusammenhang zwischen dem meteorologischen Geschehen und der Kornfeuchtigkeit hin untersucht.

\section{Kornabtrocknung}

Aus einer grossen Zahl von Messungen ergab sich, dass zumindest für diese mehr orientierenden Untersuchungen das Sättigungsdefizit um 14 Uhr für die Berechnung der Abtrocknung geeignet ist. Demgegenüber erwies sich ein Einfluss des Windes als nicht so ausschlaggebend.

Die Kurven der Abb. 1 zeigen nur einige Beispiele für den zeitlichen Verlauf der Kornabtrocknung für verschiedene Anfangsfeuchtigkeiten, mit dem Sättigungsdefizit als Parameter. Die Trocknungskurven haben einen exponentiellen Verlauf. Da in keinem Fall ein Wassergehalt unter $11 \%$ gemessen wurde, dürfte die Asymptote dieser Kurven bei 11\% Wassergehalt liegen. 


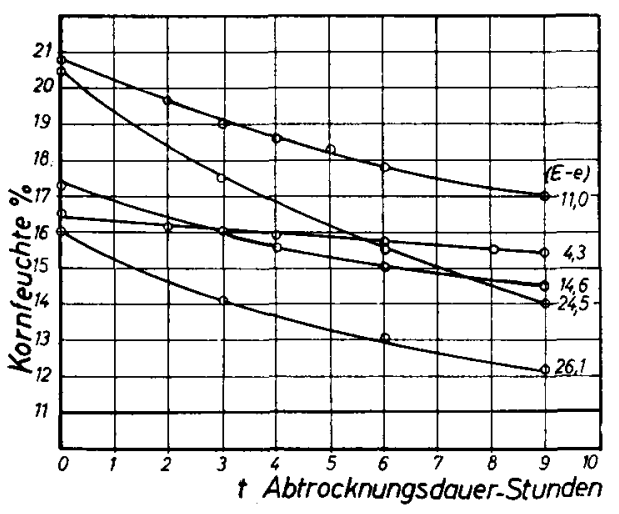

Fig. I. Zusamimenhang zWISCheN ABtrockNUNGSDAUER, SÄTTIGUNGSDEFIZIT UND KORNFEUCHTIGKEIT.

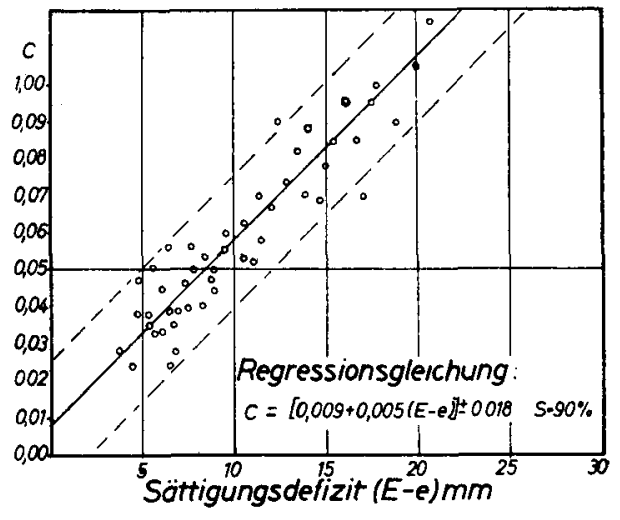

Fig. 2. Regressionsgerade für $C$ Und VERTRAUENSBEREICH FÜR $S=90 \%$.

Der Mathematische Ausdruck für diese Kurven lautet also :

$$
\left(F_{t}-11\right)=\left(F_{o}-11\right) e^{-c t}
$$

bezw. :

$$
\log \left(F_{\mathrm{t}}-11\right)=\log \left(F_{0}-11\right)-c t \log e
$$

wo $t$ die Abtrocknungsdauer in Stunden und $F_{f}$ die Kornfeuchtigkeit zur Zeit $t$ ist.

Die konstante Grösse $c$ lässt sich aus zusammengehörigen Messwerten von Anfangs- und Endfeuchtigkeit und der entsprechenden Abtrocknungsdauer errechnen.

In Abb. 2 sind die errechneten Werte $c$ in Abhängigkeit vom 14 Uhr Sättigungsdefizit, sowie die Regressionsgerade mit ihrem Vertrauensbereich für eine statistische Sicherheit von $90 \%$ eingetragen. Der Korrelationskoeffizient $r=0.9$

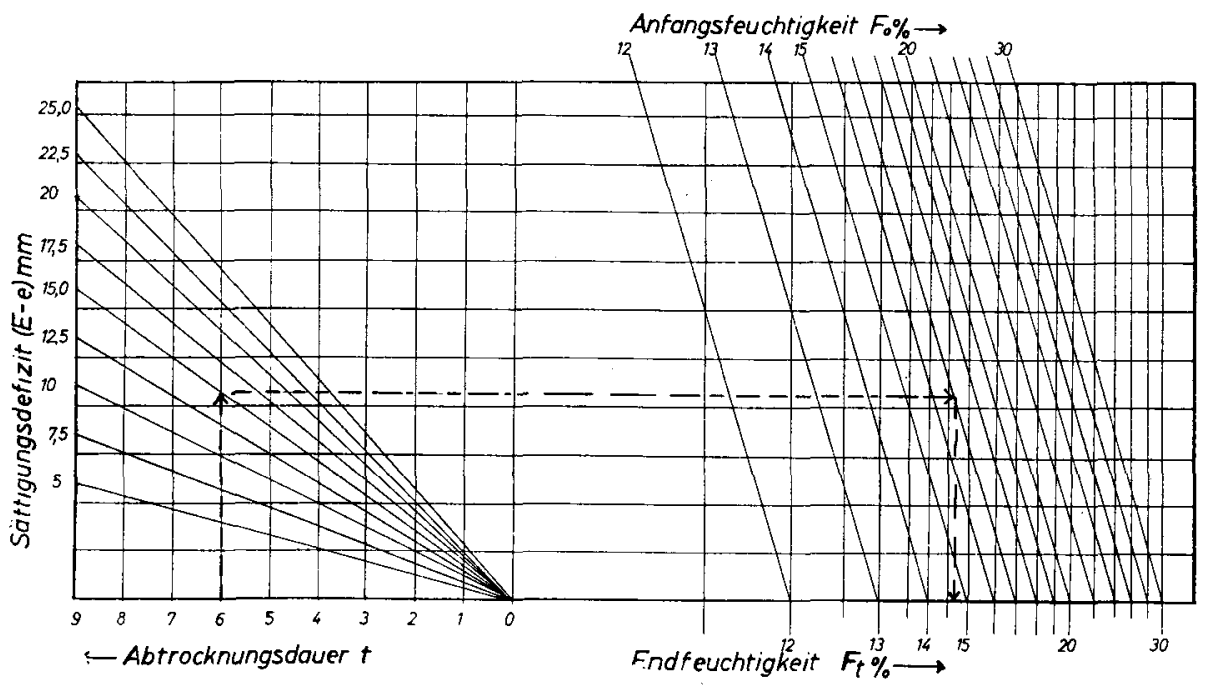

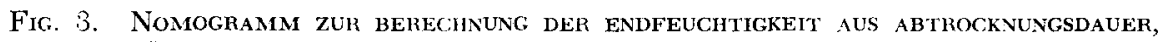
SÄTTIGUNGSDEFIZIT UND ANFANGSFEUCHTIGKEIT. 
zeigt, dass die Annahme eines linearen Zusammenhanges für praktische Zwecke mit genügender Sicherheit erlaubt ist und sich auf diesem Wege die Abtrocknung aus Sättigungsdefizit und Abtrocknungsdauer berechnen lässt. Selbst in ungünstigen Fällen dürfte der Fall höchstens 10\% der berechneten Endfeuchtigkeit betragen. Für die umfangreiche rechnerische Auswertung wurde ein Nomogramm (Abb. 3), sowie ein Sonderrechenschieber entwickelt.

\section{Kornfeuchtezunahme durch Regen und Tau}

Für die Zunahme des Wassergehaltes des Kornes durch Niederschläge ergab sich aus den Messungen der Zusammenhang:

$$
\left(33-F_{t}\right)=\left(33-F_{o}\right) e-c^{\prime} t
$$

bezw. :

$$
\log \left(33-F_{t}\right)=\log \left(33-F_{0}\right)-c^{\prime} t \log e
$$

wo $t$ die Niederschlagsdauer in Stunden bedeutet.

Trägt man die Grösse $c^{\prime} t$ über der Wurzel der Niederschlagsdauer $t$ auf, so sieht man, dass sich mit ausreichender Genauigkeit eine lineare Regression zwischen $c^{\prime} t$ und $\sqrt{t}$ ergibt.

Abb. 4 zeigt sowohl die errechnete Regressionsgerade mit ihrem Vertrauensbereich als auch das für die praktische Rechnung entwickelte Nomogramm.

Aus einer grösseren Zahl von Messungen der Feuchtezunahme durch Tau ergab sich, dass mit genügender Näherung diese sich aus dem Nomogramm für Regen entnehmen lässt, wenn man einer bestimmten Taustärke eine bestimmte Regendauer zuordnet (siehe Abb. 4).

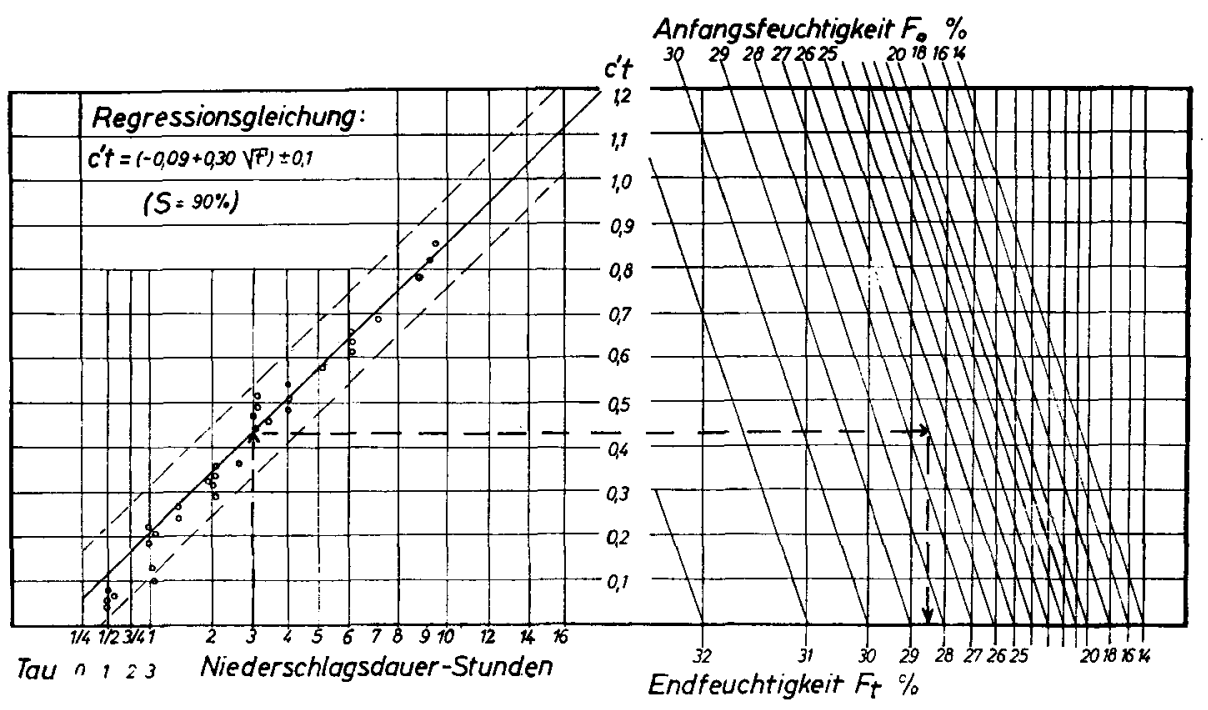

Fig. 4. . Nomogramm zUR BEREChNUNG DER ENDFEUChTtGKeIt AUS NIEDERSCHLAGSDAUER (ODER TAUSTÄRKE) UND ANFANGSFEUCHTIGKEIT.

\section{Bestimmung der Mähdreschereinsatzstunden}

Auf Grund dieser Ergebnisse wurden nun für 97 Klimastationen des Bundesgebietes für die phänologisch festgelegten Erntezeitspanne der Jahre 1946-1954 
der vermutliche Kornfeuchteverlauf errechnet. Ein Beispiel dafür bringt Abb. 5 . Als Einsatzstunden für den Mähdrescher wurden nun diejenigen mit einer Kornfeuchtigkeit unter $20 \%$ bezw. $17 \%$ ausgezählt und addiert. Die sich so im Mittel und im ungünstigsten Jahr ergebenden Mähdreschereinsatzstunden wur-

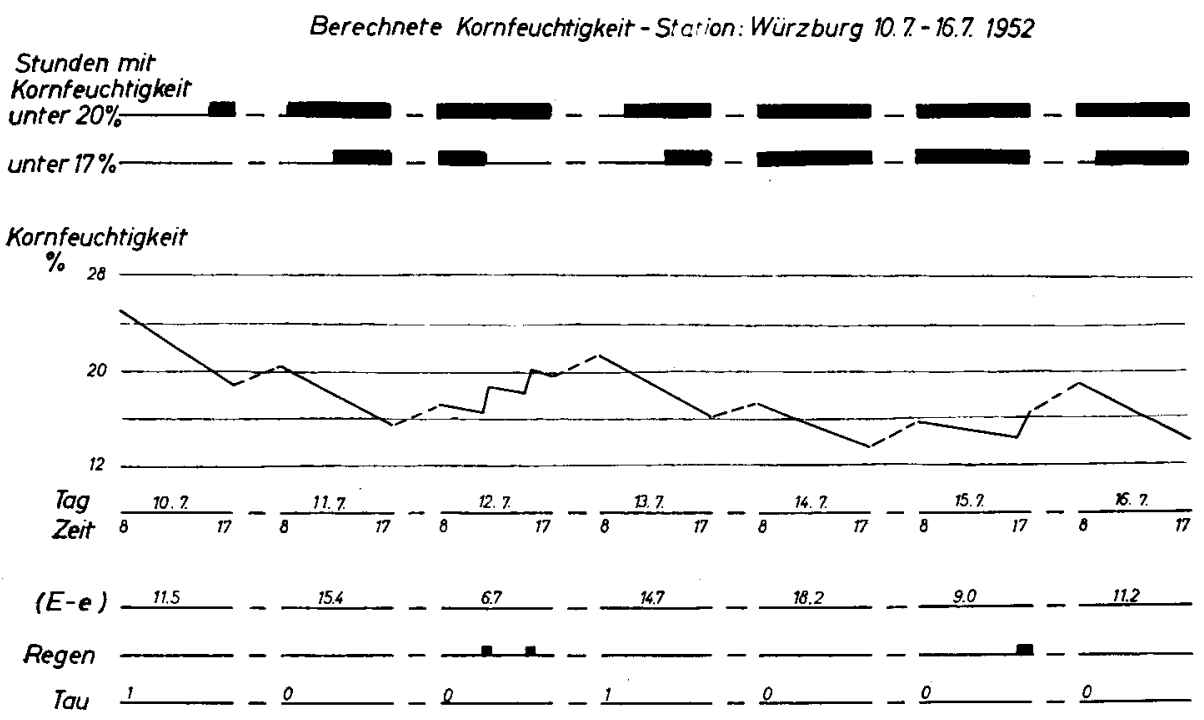

Fig. 5. BEISPIEL DER KORNFEUCHTEberechnung FÜR EINEN SPEZialfall.

den von der Agrarmeteorologischen Abteilung des Deutschen Wetterdienstes in zwei Karten eingetragen, wodurch sich zunächst ein grober Uberblick über die betriebswirtschaftlichen Möglichkeiten dieses Ernteverfahrens in den verschiedenen Gebieten ergibt.

\section{Literatur}

BerG, M. und L. Ottoson: Skördetröskning vid lag Vattenhalt. Ultuna-Uppsala Meddl. Nr. 223 (1949).

VoIGT, V. : Der Kornfeuchtigkeitsverlauf auf dem Halm stehenden Getreides unter dem Einfluss der Witterung und Folgerungen für den Mähdrusch. (Dissertation Hohenheim, erscheint demnächst). 